\title{
Pitavastatin suppressed liver cancer cells in vitro and in vivo
}

\author{
This article was published in the following Dove Press journal: \\ OncoTargets and Therapy \\ 29 August 2016 \\ Number of times this article has been viewed
}

\section{He-YiYou \\ Wei-Jian Zhang \\ Xue-Meng Xie \\ Zhi-Hai Zheng \\ Heng-Liang Zhu \\ Fei-Zhao Jiang}

Department of Telescopic Surgery, The First Affiliated Hospital of Wenzhou Medical University, Wenzhou, People's Republic of China
Correspondence: Wei-Jian Zhang Department of Telescopic Surgery, The First Affiliated Hospital of Wenzhou Medical University, Nanbaixiang Town, Wenzhou 325000, People's Republic of China

Email39209488@qq.com

\begin{abstract}
Pitavastatin classically functions as a blood cholesterol-lowering drug. Previously, it was discovered with antiglioma stem cell properties through drug screening. However, whether it can be used for liver cancer cell therapy has never been reported. In this study, the cell viability and colony formation assay were utilized to analyze the cytotoxicity of pitavastatin on liver cancer cells. The cell cycle alteration was checked after pitavastatin treatment. Apoptosis-related protein expression and the effect of caspase inhibitor were also checked. The in vivo inhibitory effect of pitavastatin on the growth of liver tumor was also tested. It was found that pitavastatin inhibited growth and colony formation of liver cancer Huh-7 cells and SMMC7721 cells. It induced arrest of liver cancer cells at the G1 phase. Increased proportion of sub-G1 cells was observed after pitavastatin treatment. Pitavastatin promoted caspase- 9 cleavage and caspase- 3 cleavage in liver cancer cells. Caspase inhibitor Z-VAD-FMK reversed the cleavage of cytotoxic effect of pitavastatin. Moreover, pitavastatin decreased the tumor growth and improved the survival of tumor-bearing mice. This study suggested the antiliver cancer effect of the old drug pitavastatin. It may be developed as a drug for liver cancer therapy.
\end{abstract}

Keywords: liver cancer, apoptosis, chemosensitive, blood cholesterol lowering drug

\section{Introduction}

Liver cancer is one of the most common cancers in the world. It was found to occur in 782,000 people in the year 2012. It led to 746,000 deaths on that year. Liver cancer mainly induced by cirrhosis resulted from hepatitis B virus (HBV), hepatitis $\mathrm{C}$ virus, or alcohol. As there is a high incidence of HBV in Asian countries, the occurrence of liver cancer is high. More than 100,000,000 people in the People's Republic of China were statistically reported as HBV carriers. This makes the prevention and curation of liver cancer difficult. ${ }^{1,2}$ Looking for an efficient drug is critically important for liver cancer therapy.

Pitavastatin is a classical drug for lowering blood cholesterol. It is a type of statin drug. In the recent years, a novel function of pitavastatin was discovered by some researchers: pitavastatin was able to inhibit the growth of cancer cells. In 2006, a study showed that pitavastatin could regulate NF- $\kappa$ B and anti-inflammation in hepatocellular carcinoma cells. ${ }^{3}$ Pitavastatin has been reported in several animal models that it correlated with various types of tumor progression. ${ }^{4-6}$ Pitavastatin could induce autophagic cell death in glioma cells and promote sensitivity of cells to radiotherapy. ${ }^{7}$ It could inhibit cell proliferation and induce cell apoptosis in cholangiocarcinoma cells as well. ${ }^{8}$ In 2014 , Jiang et $\mathrm{al}^{9,10}$ found another novel function of pitavastatin, which was discovered by US Food and Drug Administration (FDA)-approved drug screening and showed strong ability of antiglioma stem cells. However, whether 
pitavastatin could be used for liver cancer therapy has never been reported earlier. In our study, we tested the antiliver cancer ability of pitavastatin using cell viability and colony formation assays. Pitavastatin inhibited the growth of liver cancer cells in a dose-dependent and time-dependent manner. Further mechanism of pitavastatin in vitro and in vivo were investigated. As pitavastatin has already been approved by the FDA, if it is efficient for liver cancer therapy, it will be a novel approach for liver cancer therapy.

\section{Materials and methods Cell culture}

The liver cancer cell lines Huh-7 and SMMC7721 were maintained in Dulbecco's Modified Eagle's Medium (Thermo Fisher Scientific, Waltham, MA, USA) with 10\% fetal bovine serum (Thermo Fisher Scientific). The medium was supplemented with penicillin and streptomycin. Both of these cells were obtained from the Cell Bank of Chinese Academy of Sciences (Shanghai, People's Republic of China).

\section{Cell count detection}

The Huh-7 cells and SMMC7721 cells were split into 96-well dishes at 5,000 cells/well and treated with the indicated dosage of pitavastatin for 48 hours or $5 \mu \mathrm{M}$ pitavastatin for 1, 2, 4, 6 days respectively. The cells were incubated with 3-(4,5-dimethylthiazol-2-yl)-2,5-diphenyltetrazolium bromide (Beyotime, Haimen, People's Republic of China) and formed formazan in the liver cells. Formazan was dissolved in dimethyl sulfoxide (DMSO; Beyotime), and the absorbance was measured at the wavelength of $570 \mathrm{~nm}$. The cells treated with DMSO were used as a control group. The relative cell number of each group was calculated as pitavastatin-treated group/cell number in the DMSO-treated group.

\section{Colony formation}

The Huh-7 cells and SMMC7721 cells were trypsinized into single cells and split into 24-well dishes at 100 cells/well. The cells were pretreated with $0 \mu \mathrm{M}, 0.5 \mu \mathrm{M}$, or $1 \mu \mathrm{M}$ of pitavastatin and cultured for 8 days. The medium was discarded, and the cells were stained with $1 \%$ crystal violet in $20 \%$ ethanol for 1 hour. The cells were washed with water thoroughly, dried, and scanned.

\section{Cell cycle}

The Huh- 7 cells were plated in six-well dishes at $3 \times 10^{5}$ cells/ well and treated with pitavastatin at the indicated dosage for 48 hours. The cells were trypsinized into single cells, fixed with $70 \%$ ethanol at $4^{\circ} \mathrm{C}$ for 30 minutes, washed with phosphate-buffered saline thrice, and incubated with $50 \mu \mathrm{g} / \mathrm{mL}$
RNAase (Sigma-Aldrich Co., St Louis, MO, USA) for 30 minutes at $37^{\circ} \mathrm{C}$. The cells were then stained with $50 \mu \mathrm{g} / \mathrm{mL}$ of propidium iodide (Sigma-Aldrich Co.) in the dark. The data were collected using the flow cytometry machine Calibur.

\section{Western blot}

The Western blot assay was performed according to the standard protocol. The primary antibodies rabbit antiprocaspase-9, antiprocaspase-3, cleaved caspase-3, cleaved PARP, and tubulin were purchased from Cell Signaling Technology (Danvers, MA, USA). The secondary antibody was obtained from Beyotime.

\section{Mice experiments}

All the animal experiments were performed according to the National Institutes of Health's Guide for the Care and Use of Laboratory Animals. All the animal experiments were approved by the The First Affiliated Hospital of Wenzhou Medical University institutional review board. The nude mice were injected with Huh-7 cells subcutaneously. Each mouse was injected with $2 \times 10^{6}$ cells, which were pretreated with DMSO or $1 \mu \mathrm{M}$ of pitavastatin for 12 hours. The tumors of the mice were measured every 4 days and calculated as length $\times$ width $\times$ width $/ 2$.

\section{Statistical analysis}

All the data were presented as mean \pm SD. The comparison between the groups was analyzed by one-way analysis of variance. $P$-value $<0.05$ was significant. The survival curve was calculated using the Kaplan-Meier method in GraphPad Prism 5.0.

\section{Results}

\section{Pitavastatin decreased growth of liver cancer cells}

Pitavastatin (Figure 1A) is a blood cholesterol-lowering drug. It was discovered effective in antiglioma cancer. To test whether it could treat antiliver cancer as well, the liver cancer Huh-7 cells and SMMC7721 cells were treated with different dosages of pitavastatin (Figure 1B). The cells were inhibited by pitavastatin, dependent of dosage. They also showed time dependence after treated with $5 \mu \mathrm{M}$ of pitavastatin (Figure 1C). Low dosage of pitavastatin was able to prevent colony formation of liver cancer cells (Figure 1D).

\section{Pitavastatin induced apoptosis of liver cancer cells}

To investigate how pitavastatin inhibited growth of liver cancer cells, cell cycle distribution of the cells after 
A

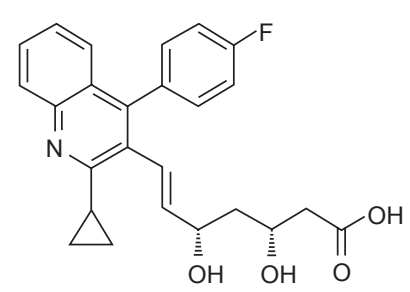

C

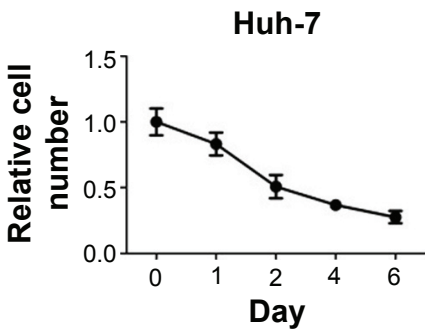

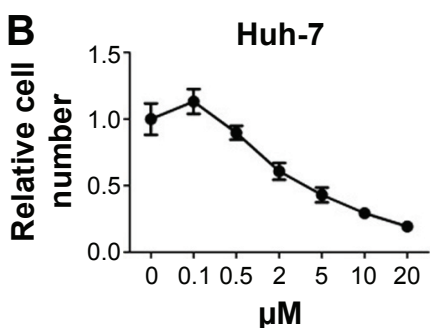
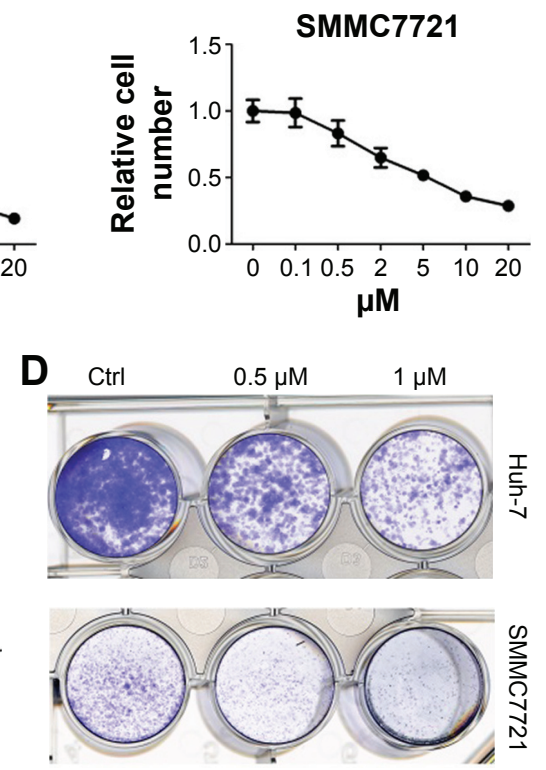

Figure I Pitavastatin decreased growth of liver cancer cells.

Notes: (A) Molecular structure of pitavastatin. (B) Cell viability detection on liver cancer Huh-7 cells and SMMC772I cells after pitavastatin treatment for 48 hours. (C) Cell viability detection on liver cancer Huh-7 cells and SMMC772I cells with $5 \mu$ M pitavastatin treatment for the indicated days. (D) Colony formation of Huh-7 cells and SMMC772I cells with pitavastatin treatment for 8 days.

Abbreviation: Ctrl, control.

pitavastatin treatment was measured. Pitavastatin dramatically arrested the Huh-7 cells at the G1 phase (Figure 2A and B). A further analysis found that pitavastatin robustly increased the proportion of sub-G1 phase cells as well as the sub-G1 phase indicated that the cells were going apoptosis (Figure 2C). Pitavastatin may inhibit the growth of liver cancer cells by both arresting the cells at the G0 phase and inducing apoptosis of cells.

\section{Pitavastatin induced apoptosis of liver cancer cells dependent of caspase}

Pitavastatin treatment increased the population of Huh-7 cells in the sub-G1 phase. It induced apoptosis of liver cancer cells. To get to know how it functioned, we measured the expression of apoptosis-related proteins. It was found that caspase- 9 and caspase- 3 as well as poly ADP ribose polymerase (PARP) were cleaved (Figure 3A and B). We used $10 \mu \mathrm{M}$ caspase inhibitor Z-VAD-FMK (Sigma-Aldrich Co.) to pretreat cells for 24 hours and then treat the cells with pitavastatin. We found that Z-VAD-FMK treatment could dramatically attenuate the effect of pitavastatin in inhibiting the growth of Huh-7 cell. This suggested that pitavastatin induced apoptosis of liver cancer cells dependent of caspases (Figure 3C).

\section{Pitavastatin prohibited liver tumor growth in vivo}

To further investigate whether pitavastatin showed in vivo inhibitory effect, drug-pretreated Huh-7 cells were injected into the rear back subcutaneously. Pitavastatin treatment decreased the growth of liver tumor in nude mice (Figure 4A). It prolonged the survival time of tumor-bearing mice as well (Figure 4B).

\section{Discussion}

Liver cancer is one of the most common cancers in the world. The most cases of liver cancer occur in Asian countries due to the high incidence of HBV infection. Prevention of liver cancer and obtaining an efficient drug for liver cancer therapy is urgent.

Pitavastatin is a small molecule. It was developed as a blood cholesterol-lowering drug many years ago. ${ }^{11,12}$ It has been reported with novel anticancer property in the recent years. However, whether it can be used for liver cancer therapy has never been reported. In this study, we tested the antiliver cancer effect of an old blood cholesterol-lowering drug pitavastatin. Pitavastatin inhibited liver cancer Huh-7 cells and SMMC7721 cells in a time-dependent and dosage-dependent manner. Pitavastatin dramatically prohibited growth and colony formation of liver cancer cells (Figure 1). We also found that pitavastatin could arrest cells at the G1 phase and induce apoptosis of liver cancer cells (Figure 2). The study published in 2011 found that statins could induce growth inhibition and apoptosis of cholangiocarcinoma cells. ${ }^{8}$ However, how it works was not well defined. To well understand this, we checked the expression level of apoptosis-related proteins and found 

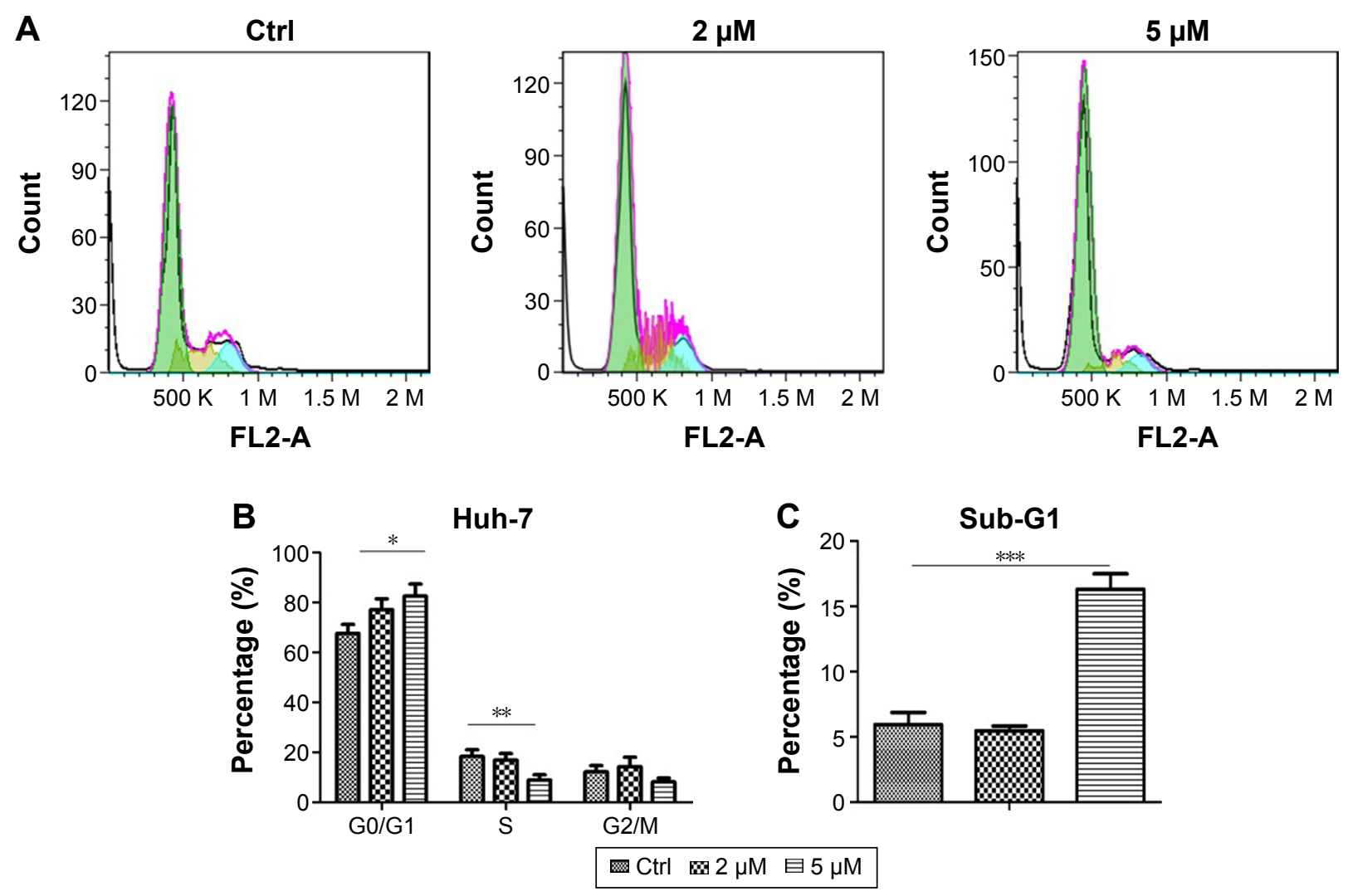

Figure 2 Pitavastatin induced apoptosis of liver cancer cells.

Notes: (A) Cell cycle distribution of Huh-7 cells after pitavastatin treatment for 48 hours. (B) Quantitative analysis of cell cycle distribution. The average of G0/GI, S, and $\mathrm{G} 2 / \mathrm{M}$ phases was calculated. (C) Quantitative analysis of sub-GI population with pitavastatin treatment. The average of sub-GI phase was calculated. $* P<0.0$ I, $* * P<0.05$, and $* * * P<0.001$

Abbreviation: Ctrl, control.

that procaspase-9 and procaspase-3 were robustly cleaved after pitavastatin treatment. Z-VAD-FMK, an inhibitor to all caspase proteins, dramatically inhibited pitavastatin-induced cell growth inhibition. This suggested that pitavastatin inducing apoptosis of liver cancer cells was dependent of caspase proteins (Figure 3). The in vivo data further confirmed the efficiency of pitavastatin on liver cancers. It prolonged the survival of tumor-bearing mice (Figure 4). In the in vivo experiments, the cells were pretreated with $1 \mu \mathrm{M}$ of pitavastatin. At this dosage in vitro for 12 hours, the Huh-7 cells were almost viable. As seen in Figure 1C, the Huh-7 cells treated with $5 \mu \mathrm{M}$ of pitavastatin for 24 hours are still with $>80 \%$ viable cells. The Huh- 7 cells responded to the pitavastatin treatment in a time- and dosage-dependent manner. Pitavastatin is an old drug lowering the blood cholesterol. It exerts multieffects in vivo, especially on the liver. In our study, we found that pitavastatin showed cytotoxicity on liver tumor cells in vitro and tumor inhibition in vivo. In this study, it is unclear whether the antitumor effect in vivo is solely induced by the cytotoxicity of pitavastatin or also affected by pitavastatin through changing the cell metabolism indirectly in vivo.
In the previous studies, pitavastatin was also discovered with other functions. Through FDA drug screening, Jiang et $a l^{10}$ found that pitavastatin could dramatically inhibit glioma cancer stem cells. This was further confirmed in another article published by this group. ${ }^{9}$ The developing of novel function of old drug, especially, the thioridazine, was also found in other researches. It is a dopamine receptor antagonist and was found with anticancer stem cells ability through drug screen. ${ }^{13}$ It was then further tested in other studies. ${ }^{14,15}$ In this study, we found that pitavastatin arrested cells at the G1 phase. This may also be functioned in liver cancer stem cells. This needs to be further studied in the future work. Another article disclosed that pitavastatin is able to alter the expression of drug-resistance genes. ${ }^{16,17}$ As one of the key characteristics of cancer stem cells is chemoresistance, pitavastatin possibly could sensitize cancer stem cells to chemotherapy by downregulating the multiple drug-resistant genes. Moreover, pitavastatin could sensitize cancer cells to radiotherapy as well. ${ }^{7}$ This further indicated the possibility of pitavastatin in promoting cancer stem cells to traditional therapies. Owing to the sensitization of cancer cells to treatment by pitavastatin, it could be combined with 
A

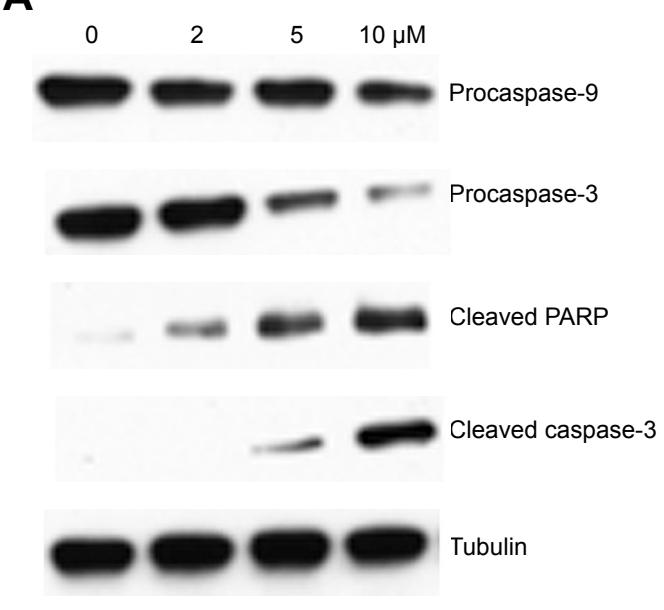

C

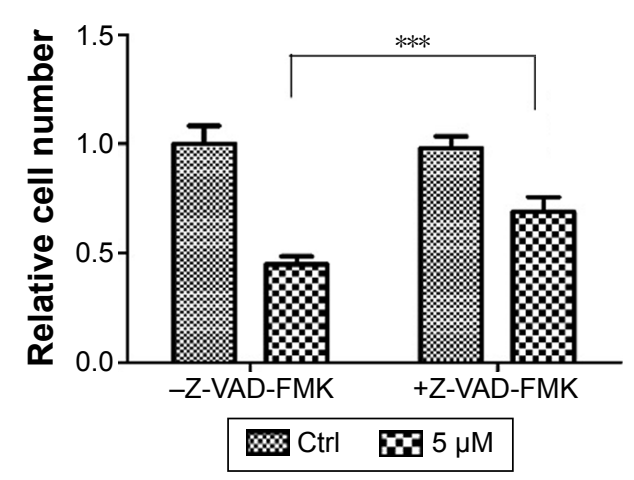

B
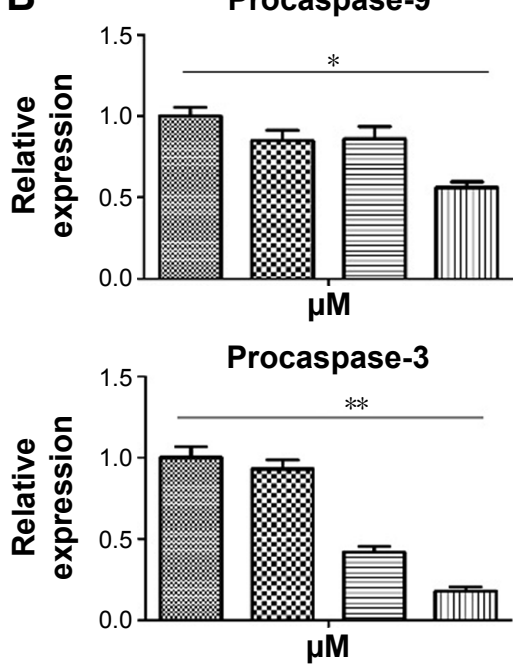

Cleaved PARP

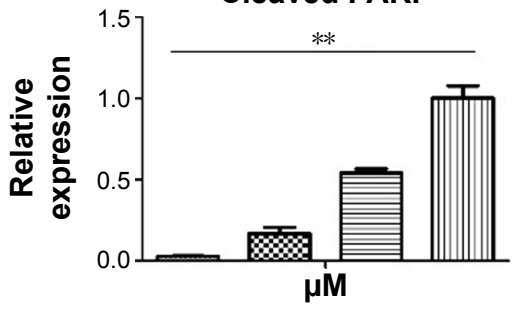

Cleaved caspase-3

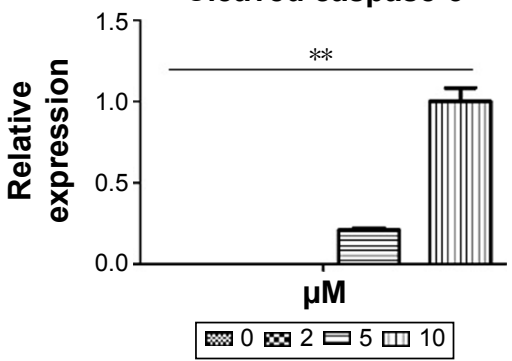

Figure 3 Pitavastatin induced apoptosis of liver cancer cells dependent of caspase.

Notes: (A) Alteration of apoptosis-related protein expression after pitavastatin treatment for 48 hours. (B) Quantitative analysis of protein expression with pitavastatin treatment. Huh-7 cells without treatment were used as a Ctrl. The relative expression was presented as pitavastatin-treated cells/DMSO-treated cells. (C) Reversal effect of caspase inhibitor Z-VAD-FMK on pitavastatin prohibited cell growth. Huh-7 cells without treatment were used as a Ctrl. The relative expression was presented as pitavastatin- or Z-VAD-FMK-treated cells/DMSO-treated cells. $* P<0.05$, $* * P<0.00$ I, and $* * * P<0.01$.

Abbreviations: Ctrl, control; DMSO, dimethyl sulfoxide.
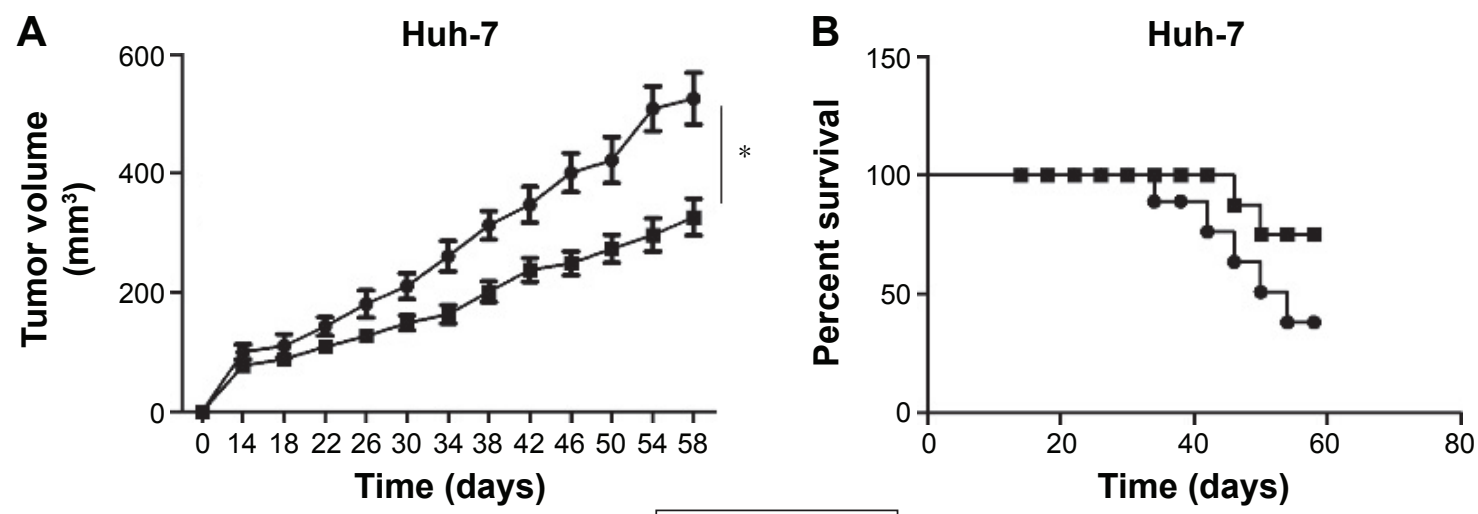

Figure 4 Pitavastatin prohibited liver tumor growth in vivo.

Notes: (A) Tumor growth curve of Huh-7 cells with or without pitavastatin treatment. (B) Mice survival outcome with or without pitavastatin treatment. $* P<0.00$ I. Abbreviation: Ctrl, control. 
other treatment to enhance its efficiency on cancer treatment. Chemotherapeutic drug, radiation, traditional Chinese medicine, small molecules, or viruses can be used for this combination. ${ }^{18-20}$ The famous small molecule resveratrol, extracted from grape, was widely used for cancer therapy in various types of cancers. ${ }^{21}$ It was used to treat cancer cells or cancer stem cells, together with other approaches. ${ }^{22,23}$ Whether pitavastatin could also target liver cancer stem cells will be further investigated in our future study. Its combination with cisplatin, fluorouracil, and oncolytic viruses may show excellent antiliver cancer cells effect. In this study, our results suggested pitavastatin as an effective molecule for liver cancer therapy. It could be considered as a potential drug for liver cancer therapy in the future.

\section{Disclosure}

The authors report no conflicts of interest in this work.

\section{References}

1. Siegel R, Naishadham D, Jemal A. Cancer statistics, 2012. CA Cancer J Clin. 2012;62(1):10-29.

2. Torre LA, Bray F, Siegel RL, Ferlay J, Lortet-Tieulent J, Jemal A. Global cancer statistics, 2012. CA Cancer J Clin. 2015;65(2):87-108.

3. Wang J, Tokoro T, Higa S, Kitajima I. Anti-inflammatory effect of pitavastatin on NF-kappaB activated by TNF-alpha in hepatocellular carcinoma cells. Biol Pharm Bull. 2006;29(4):634-639.

4. Kubatka P, Bojkova B, Kassayova M, et al. Combination of Pitavastatin and melatonin shows partial antineoplastic effects in a rat breast carcinoma model. Acta Histochem. 2014;116(8):1454-1461.

5. Miyamoto S, Yasui Y, Kim M, et al. A novel rasH2 mouse carcinogenesis model that is highly susceptible to 4-NQO-induced tongue and esophageal carcinogenesis is useful for preclinical chemoprevention studies. Carcinogenesis. 2008;29(2):418-426.

6. Sata M, Nishimatsu H, Osuga J, et al. Statins augment collateral growth in response to ischemia but they do not promote cancer and atherosclerosis. Hypertension. 2004;43(6):1214-1220.

7. Tsuboi Y, Kurimoto M, Nagai S, et al. Induction of autophagic cell death and radiosensitization by the pharmacological inhibition of nuclear factor-kappa B activation in human glioma cell lines. J Neurosurg. 2009;110(3):594-604.

8. Kamigaki M, Sasaki T, Serikawa M, et al. Statins induce apoptosis and inhibit proliferation in cholangiocarcinoma cells. Int J Oncol. 2011;39(3): 561-568.
9. Jiang $\mathrm{P}$, Mukthavaram R, Chao $\mathrm{Y}$, et al. In vitro and in vivo anticancer effects of mevalonate pathway modulation on human cancer cells. Br J Cancer. 2014;111(8):1562-1571.

10. Jiang $P$, Mukthavaram R, Chao $Y$, et al. Novel anti-glioblastoma agents and therapeutic combinations identified from a collection of FDA approved drugs. $J$ Transl Med. 2014;12:13.

11. Teramoto T, Shimano H, Yokote K, Urashima M. Effects of pitavastatin (LIVALO Tablet) on high density lipoprotein cholesterol (HDL-C) in hypercholesterolemia. J Atheroscler Thromb. 2009;16(5):654-661.

12. Kajinami K, Takekoshi N, Saito Y. Pitavastatin: efficacy and safety profiles of a novel synthetic HMG-CoA reductase inhibitor. Cardiovasc Drug Rev. 2003;21(3):199-215.

13. Sachlos E, Risueno RM, Laronde S, et al. Identification of drugs including a dopamine receptor antagonist that selectively target cancer stem cells. Cell. 2012;149(6):1284-1297.

14. Min KJ, Seo BR, Bae YC, Yoo YH, Kwon TK. Antipsychotic agent thioridazine sensitizes renal carcinoma Caki cells to TRAIL-induced apoptosis through reactive oxygen species-mediated inhibition of Akt signaling and downregulation of Mcl-1 and c-FLIP(L). Cell Death Dis. 2014;5:e1063.

15. Mu J, Xu H, Yang Y, et al. Thioridazine, an antipsychotic drug, elicits potent antitumor effects in gastric cancer. Oncol Rep. 2014;31(5): 2107-2114.

16. Yue W, Lee JK, Abe K, Sugiyama Y, Brouwer KL. Decreased hepatic breast cancer resistance protein expression and function in multidrug resistance-associated protein 2-deficient (TR(-)) rats. Drug Metab Dispos. 2011;39(3):441-447.

17. Arii K, Suehiro T, Ota K, et al. Pitavastatin induces PON1 expression through p44/42 mitogen-activated protein kinase signaling cascade in Huh7 cells. Atherosclerosis. 2009;202(2):439-445.

18. Tao C, Yang LX. Improved radiotherapy for primary and secondary liver cancer: stereotactic body radiation therapy. Anticancer Res. 2012; 32(2):649-655.

19. Nelson RL, Freels S. A systematic review of hepatic artery chemotherapy after hepatic resection of colorectal cancer metastatic to the liver. Dis Colon Rectum. 2004;47(5):739-745.

20. Mu J, Liu T, Jiang L, et al. The traditional Chinese medicine baicalein potently inhibits gastric cancer cells. J Cancer. 2016;7(4):453-461.

21. Baur JA, Sinclair DA. Therapeutic potential of resveratrol: the in vivo evidence. Nat Rev Drug Discov. 2006;5(6):493-506.

22. Yang Y, Xu H, Huang W, et al. Targeting lung cancer stem-like cells with TRAIL gene armed oncolytic adenovirus. J Cell Mol Med. 2015; 19(5):915-923.

23. Nessa MU, Beale P, Chan C, Yu JQ, Huq F. Combinations of resveratrol, cisplatin and oxaliplatin applied to human ovarian cancer cells. Anticancer Res. 2012;32(1):53-59.
OncoTargets and Therapy

\section{Publish your work in this journal}

OncoTargets and Therapy is an international, peer-reviewed, open access journal focusing on the pathological basis of all cancers, potential targets for therapy and treatment protocols employed to improve the management of cancer patients. The journal also focuses on the impact of management programs and new therapeutic agents and protocols on

\section{Dovepress}

patient perspectives such as quality of life, adherence and satisfaction. The manuscript management system is completely online and includes a very quick and fair peer-review system, which is all easy to use. Visit $\mathrm{http}: / /$ www.dovepress.com/testimonials.php to read real quotes from published authors. 